\title{
PATRONES DE AUTORIDAD E INFLUENCIA EN INSTAGRAM PARA LA GESTIÓN DE LA COMUNICACIÓN ORGANIZACIONAL Y DE LAS MARCAS PERSONALES
}

\section{Sixto García, José ${ }^{1}$ \\ Universidad de Santiago de Compostela, España \\ jose.sixto@usc.es}

Material original autorizado para su primera publicación en la revista académica REDMARKA. Revista Digital de Marketing Aplicado.

https://doi.org/10.17979/redma.2018.01.021.4840

Recibido: 15 enero 2018

Aceptado: 8 agosto 2018

\section{Resumen}

Con más de 800 millones de usuarios activos al mes Instagram se ha convertido en la red de moda para la distribución de contenido audiovisual, especialmente fotografía y vídeo corto, las denominadas historias. La pertinencia de la red para la explotación de las marcas personales la demuestran los ejemplos de influencers que han nacido en Instagram y que ahora actúan como prescriptores de otras marcas comerciales. Sin embargo,

\footnotetext{
${ }^{1}$ Santiago de Compostela (España). Director de Instituto de Medios Sociales (Cidade da Cultura de Galicia) y profesor asociado de la Universidade de Santiago de Compostela. Doctor en Comunicación y Periodismo con Mención Europea y Premio Extraordinario, Máster MBA en Dirección y Gestión de Empresas, Máster en Formación de Profesorado y Licenciatura en Periodismo, también con Premio Extraordinario. Autor de libros como Fundamentos de Marketing Digital, Las redes sociales como estrategia de marketing online o Marketing y Comunicación, y también de estudios y artículos científicos en revistas de impacto como, por ejemplo, el último análisis sobre la Presencia 2.0 de las PYMES gallegas, publicado en la Revista Latina de Comunicación Social. Trabajó como director de Marketing y Comunicación en el IECS y ha impartido numerosas formaciones en empresas, Cámaras de Comercio e instituciones varias.
} 
¿cómo pueden aprovechar las organizaciones esta red para acercarse a los públicos y mejorar los niveles de engagement?

En este texto analizamos las posibilidades y los recursos que ofrece Instagram para la gestión de la comunicación en las organizaciones, diferenciando entre las prácticas propias de usuarios particulares y las estrategias orientadas a un tratamiento profesional de la marca. Se presta especial atención a las potencialidades del contenido autodestruible que la red permite materializar a través de las historias para determinar su pertinencia de uso en la planificación de community management. Se describen y analizan los patrones y los modelos de gestión que denotan autoridad e influencia y se examina qué cuentas alcanzan mayor notoriedad en la red. Se concluye valorando los estándares de analítica que se han de considerar para la medición de audiencias, del retorno y de la inversión realizada.

Palabras clave: Instagram, comunicación organizacional, historias, contenido autodestruible, influencia, personal branding

\section{Abstract}

With more than 800 million active users every month, Instagram has become the fashion network for the distribution of audiovisual content, especially photography and short video. The relevance of the network for the exploitation of personal brands is demonstrated by the examples of influencers that were born on Instagram and now act as prescribers of other commercial brands. However, how can organizations take advantage of this network to get closer to the public and improve engagement levels?

In this paper we analyze the possibilities and resources offered by Instagram for the management of communication in organizations, differentiating between the practices of particular users and the professional treatment of a brand. Special attention is paid to the potential of the self-destructive content (Instagram stories) and its relevance for using in community management planning. We 
describe and analyze the patterns and management models that denote authority and influence so, on the other hand, the most notoriety accounts in the network are examined. It is concluded by evaluating the analytical standards that must be considered for the measurement of audiences, of the return and of the investment made.

Keywords: Instagram, Organizational Communication, stories, self-destructive content, influence, personal branding

\section{CONTEXTUALIZACIÓN}

Los usuarios de las redes sociales están cada vez más interesados en las imágenes y cada vez menos en los textos (Mamani \& al., 2017; García, Lópezde-Ayala \& Catalina, 2013). Es más rápido y más cómodo ver que leer, por eso los contenidos audiovisuales se han convertido en imprescindibles en las redes sociales horizontales o generalistas, y no solo en aquellas cuyo modelo de funcionamiento se sustenta precisamente en este tipo de contenido como es el caso de Instagram o YouTube.

La evolución de las nuevas formas de comunicación resulta determinante en la transformación de ideas, valores y formas de comportamiento de los usuarios (Garmendia, Garitaonandia \& al., 2012; Patchin \& Hinduja, 2010; Bandura, 2006; Bazanini, 2015), de tal forma que sería un sinsentido que las marcas no valorasen el comportamiento y los hábitos de consumo del público (Planells, 2017; Mullo-Lopez \& Yaguana-Romero, 2017) y, al mismo tiempo, sería ingenuo pensar que las audiencias no esperarán de las marcas las mismas prácticas comunicativas a las que están acostumbradas en las relaciones con sus contactos personales.

Sin embargo, no se trata solo de una cuestión considerable desde el punto de vista de los paradigmas comunicativos, sino también en términos de 
rentabilidad empresarial. En efecto, como estos contenidos son los que más interesan a las audiencias son los que más visualizaciones e interacciones sociales consiguen (Rodriguez \& al., 2017) y, por tanto, los más rentables desde la óptica de la gestión organizacional. Si detrás de la mayoría de estrategias de community management se esconde la intención de alcanzar el mayor número de usuarios posibles para lograr autoridad e influencia entre los usuarios (Horta, Rodrigues \& Dos Santos, 2018), parece lógico que, luego, esos prosumers aviven y redifundan los contenidos que les resultan atractivos y que acaban generando viralidad per se.

El contenido audiovisual sirve para atraer visitas a la web corporativa desde las redes sociales, aumenta el reconocimiento de marca y posibilita la obtención de opiniones de consumo (Alp \& Oguducu, 2018). Pero no todo vale y, por ello, para garantizar su contribución a los estándares de autoridad e influencia se ha de trabajar esmeradamente el storyboard y establecer con claridad cuál es la finalidad por la que la organización ha decidido incorporar el vídeo en su estrategia de medios sociales.

El videomarketing, o utilizar vídeo en las estrategias de marketing y comunicación, es, antes de nada, una manera de diferenciarse de la competencia, pues no todas las organizaciones lo utilizan a pesar de que los costes de producción se han reducido y los gastos de distribución casi no existen debido al poder viral de sitios como YouTube o Vimeo. El vídeo genera una confianza y una credibilidad difíciles de igualar y, además, crea una atmósfera de empatía y cercanía que envuelve al usuario (Engelstaetter \& Ward, 2018). Además, contribuye a aumentar los tiempos medios de duración de las visitas (autoridad) y, por tanto, a mayor duración de visita, mayor probabilidad de conversión (influencia).

Al igual que las meras presencias en redes sociales tienen un efecto anecdótico y residual en las políticas de comunicación -incluso 
contraproducente-, el uso del vídeo debe responder a una necesidad real de la organización (Gupta \& al., 2018) y no a una moda. Los modelos actuales priman cada vez más las historias que humanizan la marca (Gruner \& Power, 2018; Godwin, Khan \& Yellowlees, 2017) en detrimento de aquellos que tienen apariencia de spot publicitario. Algunos ejemplos que argumentan la pertinencia de acciones de videomarketing serían los siguientes:

a) Vídeos tutoriales o educativos con finalidad didáctica.

b) Vídeos de uso de un producto para estimular la imaginación de los compradores que se vislumbran utilizándolo.

c) Vídeos testimoniales que recogen la opinión de usuarios y transmiten confianza.

d) Vídeos de bienvenida o presentación de la organización. En este modelo cada vez cobran más importancia los empleados y menos los directivos debido a la tendencia contemporánea de humanizar las marcas.

e) Vídeos sobre proyectos realizados que evidencian la trayectoria de la organización y transmitan seguridad a los nuevos stakeholders.

f) Videoblogs. Son frecuentes en la explotación de marcas personales.

g) Vídeos que los seguidores envían para participar en un concurso organizado por la empresa y que los hace sentir partícipes sin ser conscientes de que son ellos los que están creando un contenido que luego utilizará la organización.

h) Vídeos que retransmiten en directo determinados eventos.

i) Vídeos de contenido autodestruible vinculados a situaciones más informales.

En paralelo a la expansión de estos esquemas de viralidad y propagación de los contenidos audiviosuales han surgido nuevos perfiles profesionales que actúan como difusores potenciales de contenidos de terceros o como prescriptores de marcas que canalizan y recomiendan entre su comunidad de seguidores. Estas figuras influencers, que son mucho más comunes e 
'influyentes' en las redes de componente audiovisual que en las otras (De Veirman \& al., 2017) gozan de gran credibilidad entre sus followers y, por tanto, la autoridad e influencia que ejercen sobre ellos los han convertido en valiosos embajadores de marcas comerciales que desean explorar o potenciar determinados nichos de mercado. De hecho, instagramers y youtubers son ya un elemento fundamental en las acciones de marketing digital que practican muchas organizaciones (Abidin, 2016; Agustina \& Steinberg, 2017), que han visto en el sistema de recomendaciones de estas figuras un canal de distribución excelente para los productos que ofrecen.

En las redes sociales generalistas y no sustentadas en los contenidos audiovisuales la figura del influencer no es ni tan relevante ni tan apreciable como en Instagram o en YouTube, salvo en Twitter, aunque los campos de especialización sobre los que se le presupone autoridad también difieren. Si en Twitter la influencia se manifiesta en temas políticos o en áreas concretas del conocimiento, en las redes audiovisuales la moda, la imagen, la estética, el fitness, la música, la peluquería, la nutrición o el mundo del corazón, entre otros, han encontrado su segmento de mercado. Cierto es, por otra parte, que los usuarios de este tipo de redes son en su mayoría mucho más jóvenes (IAB, 2017) y, a medida que aumenta la edad del usuario, se complica la capacidad de influir en él, pues su experiencia de vida y de adquisiciones anteriores le ayudan a conformar una opinión de consumo más sólida y menos manipulable.

Desde su creación en 2010, Instagram ha ido evolucionando hasta convertirse en la red social de moda y en líder indiscutible de las redes que sirven para compartir fotografías, todavía más desde que asumió el modelo narrativo que permite compartir historias efímeras (Instagram stories) e inundarlas de numerosos iconos, ideogramas, emojis, stickers y filtros. Estos formatos snap tienen su origen en Snapchat, una red social surgida también en 2010 y que se caracteriza por facilitar la emisión de mensajes audiovisuales de carácter efímero y autodestruible. No obstante, aunque el nacimiento de este modelo 
narrativo esté ligado a Snapchat, es precisamente en las redes de la competencia donde ha ganado más popularidad y se ha convertido en el fenómeno que es hoy en día (Sixto, 2017).

Instagram supera ya la barrera de los 800 millones de usuarios y se consolida como la tercera red social con más usuarios en el mundo, solo por detrás de Facebook -que cuenta con 2.167 millones- y YouTube -que alcanza los 1.500 millones- (Galeano, 2018) ${ }^{2}$. Es una red en la que prima la imagen -estática o en movimiento- sobre cualquier otro tipo de contenido, en especial sobre el texto, que prácticamente no se concibe sin la utilización de hashtags. Ha ido progresando hacia la filosofía de red que permite compartir la vida en vivo y en directo, y eso hace que sea mucho más que una red para compartir fotografías, hasta el punto de que, desde la óptica empresarial, es una de las redes que mayor engagement consigue con los públicos.

\subsection{Instagram en la gestión de marcas}

La pertinencia de utilizar Instagram en la gestión de marcas personales o comerciales es una decisión que corresponde al community manager en función de la política comunicativa de la entidad y de su aportación a las estrategias proyectadas en el Plan de Medios Sociales. Sin embargo, el community no puede desconsiderar la popularidad que la red ha conseguido entre las audiencias y entre las organizaciones de la competencia, pues cada vez son más las que la utilizan.

El primer factor que determina la oportunidad de uso de Instagram en la comunicación organizacional consiste en valorar inexcusablemente si se tiene material para publicar. No todas las organizaciones pueden disponer de

\footnotetext{
${ }^{2}$ Facebook e Instagram pertenecen al mismo grupo empresarial, por lo que en total concentran 2.967 millones de usuarios registrados. A ellos habría que añadir los 1.300 millones de usuarios de WhatsApp, que también forma parte del mismo grupo, lo que suma 4.267 millones de usuarios registrados. Ha de tenerse en cuenta, no obstante, que no se trata de usuarios exclusivos, pues un mismo usuario puede estar registrado en más de un sitio a la vez.
} 
contenido audiovisual suficiente para nutrir una red de este tipo. Al igual que no tiene sentido tener un canal de YouTube si no disponemos de vídeos corporativos, es absurdo crear una cuenta en Instagram si no contamos con el material audiovisual necesario. En segundo lugar, el modelo de storytelling de Instagram es fundamentalmente móvil tanto por las funciones de geolocalización que incorpora como por su propia idiosincrasia, puesto que para contar la vida en directo se requiere contarla desde el lugar en que sucede.

Por último, la incorporación de una red al Plan de Medios Sociales no solo está determinada por sus potencialidades y recursos comunicativos, sino también por el grado de popularidad que alcanza entre los públicos. En este sentido, aparte del número de usuarios activos -que está científicamente avalado por valores absolutos-, se ha de considerar la interacción que generan esos usuarios, pues importa tanto el volumen de usuarios como su comportamiento activo y colaborativo en la red. Instagram es una red muy apetecible para las empresas, y eso lo saben ellas y lo sabe Instagram. Por eso, ofrece la opción de crear perfiles de empresa que resultan la única presencia válida para las marcas que deseen participar.

Los perfiles de empresa aportan más funcionalidades que los perfiles personales, pues están concebidos para ser gestionados profesionalmente y nunca con un uso particular. Así, permiten incluir más información para que al usuario le resulte más fácil y cómodo contactar con la organización (teléfono, correo electrónico, 'cómo llegar'), posibilitan la opción de promocionar las publicaciones y garantizan el acceso a los datos estadísticos de medición de audiencias e interacción social.

Las estadísticas solo están disponibles en la app, lo que ratifica la necesidad de emplear algún tipo de dispositivo móvil para la gestión de esta red. Aparte del número de visitas que recibe el perfil en los últimos siete días, las 
estadísticas ofrecen otros datos relevantes que es necesario considerar para comprobar el cumplimiento de objetivos y adecuar la estrategia a las necesidades de la organización: (1) evolución del número de seguidores; (2) evolución del número de impresiones de las publicaciones; (3) información sobre el perfil de público: sexo, intervalos de edad y principales lugares desde los que se consulta el perfil corporativo; (4) información sobre el comportamiento de los seguidores en cuanto a promedios de horas que pasan al día en Instagram, así como los días en los que son más activos; (5) retorno de las publicaciones promocionadas en cuanto a impresiones (número total de veces que los usuarios han visto las publicaciones), alcance (número de cuentas únicas que han visto alguna de las publicaciones corporativas), visitas al perfil y seguidores; (6) audiencia de las historias en cuanto a impresiones, cantidad de espectadores y respuestas e, incluso, número de usuarios que han deslizado el dedo para descartar la historia. Al igual que en el caso de las historias que se realizan desde los perfiles personales, se puede comprobar qué usuario en concreto visualizó el contenido. Es la audiencia con nombre y apellidos, algo que otras redes todavía no ofrecen.

Instagram también pone a disposición de las organizaciones su propio sistema de promociones. Es un sistema básico e intuitivo, articulado en torno a tres objetivos prioritarios: (1) obtener más visitas al perfil de empresa; (2) conseguir más visitas al sitio web corporativo; (3) llegar a personas cerca de una ubicación. En los tres casos la red sugiere los botones de call to action para interpelar al usuario y redirigirlo a una landing page. Una vez realizada una publicación se podrá elegir la opción de promocionarla, configurándola según el público objetivo al que se quiere llegar, el presupuesto destinado y la duración establecida. 


\subsection{Instagram stories}

Las historias de Instagram encajan en lo que podemos denominar formatos snap, caracterizados por ser contenidos audiovisuales que se autodestruyen en un período máximo de 24 horas (efímeros y autodestruibles) y que, debido a su popularidad y frescura, resultan una opción excelente para el storytelling personal o corporativo (Seyfi \& Soydas, 2017). Podríamos considerarlos como la evolución de los típicos estados en texto en las redes sociales y otros sistemas 2.0 a contenido audiovisual. Las grandes redes han apreciado el potencial de un recurso que utilizaba una red más modesta (Snapchat) y asimilaron el modelo para incorporarlo a la oferta comunicativa que ofrecen a los usuarios. Después de siete años de evaluación del formato, Instagram, Facebook y WhatsApp -todos pertenecen al mismo grupo empresarialdecidieron incorporar esta modalidad de comunicación en 2017; en los dos primeros casos, decidieron llamarlas historias y, en el tercero, actualizaciones de estado (Sixto, 2017).

Entre las características, recursos y potencialidades que ofrecen las historias de Instagram para las organizaciones encontramos las siguientes:

1) Posibilidades de presentación: las historias permiten compartir contenidos audiovisuales en forma de foto, vídeo o imágenes en bucle, por lo que están claramente comprometidas con el contenido organizacional. Por otra parte, permiten agregar dibujos, texto, filtros, pegatinas, gifts o emojis para adornar la producción. Es el paso del estado en texto al estado audiovisual.

2) Duración: las historias duran aproximadamente 10 segundos, lo que necesariamente implica la primacía del impacto visual y el call to action.

3) Contenido autodestruible: todas las historias desaparecen a las 24 horas sin posibilidad de que la red de contactos del usuario vuelva a 
visualizar ese contenido. Prima, por lo tanto, lo efímero sobre lo permanente.

4) Permanencia: las historias, a diferencia de las publicaciones o la información compartida, no dejan rastro en el perfil de los usuarios ni de las empresas porque desaparecen para siempre -a no ser que se añadan al perfil como historias destacadas-. Su carácter perecedero permite publicar lo que no es apropiado para permanecer en el perfil, pero que al mismo tiempo puede despertar interés en los públicos.

5) Transmisión en vivo: aunque las historias no requieren necesariamente transmisión en vivo, esa es su auténtica filosofía porque están diseñadas para contar hechos en el mismo momento en que están sucediendo o, al menos, en un periodo de tiempo muy próximo a cuando ocurrió el acontecimiento. Es el paso de la comunicación móvil a la comunicación en vivo y en directo.

6) Plus de la innovación: Los formatos snap son un elemento incipiente en la comunicación móvil y digital, por lo que su mera práctica ya supone un plus de innovación que aporta valor añadido a la organización.

7) Opción de añadir algunas de las historias como historias destacadas en el perfil, lo que permite conservar algunos momentos relevantes sin que se autodestruyan. Se puede elegir una imagen de portada y nombre para cada historia destacada.

8) Feedback y audiencia: una de las innovaciones más notables de las historias con respecto al tipo de contenido que podía ser compartido hasta el momento es la posibilidad real y fidedigna, asegurada por el servidor de Instagram, de comprobar la lista de usuarios que visualizaron el contenido. Desde el punto de vista de la comunicación organizacional, esto permite a las entidades obtener un informe analítico de la audiencia en tiempo real y enfocar la producción 
informativa siguiendo parámetros de segmentación cada vez más precisos y efectivos.

9) Posibilidad de incorporar un link en la historia mediante la opción de 'ver más'. Esta posibilidad solo está disponible para las cuentas que se consideran influyentes, es decir, las que poseen un mínimo de 10.000 seguidores.

Así pues, las marcas que emplean Instagram en la gestión de su estrategia comunicativa no deben desaprovechar uno de los recursos con más auge en la comunicación actual, que implica un nuevo modelo de storytelling narrativo al que los usuarios ya se han acostumbrado y que, por tanto, también esperan de las marcas a las que siguen o les interesan. Emplear este tipo de formatos en el ejercicio habitual de la comunicación organizacional implica aprovechar un canal más para favorecer el engagement con los públicos y establecer con ellos un diálogo todavía más bidireccional e interactivo, donde cada vez es más relevante la narración de los hechos en el mismo momento en que se producen, lo que acentúa y potencia el call to action, la autoridad, la influencia y la primacía del contenido audiovisual e interactivo frente al estático e inmóvil.

La mayoría de las historias que se difunden en Instagram reproducen más momentos de la vida personal que contenidos corporativos, algo que, por otra parte, es lógico, pues el número de usuarios particulares es muy superior al número de organizaciones ${ }^{3}$. Sin embargo, no podemos desconsiderar el potencial del formato para las marcas, que, además de los mecanismos autónomos de publicación, pueden ofrecer contenido patrocinado a usuarios que no las siguen, pero que son seleccionados por el algoritmo de la red social en función de los criterios de elección de los públicos más afines.

\footnotetext{
${ }^{3}$ En un estudio anterior (Sixto, 2017) se comprobó que la mayoría de las historias que se difunden en Instagram reproducen momentos de la vida personal $(94,91 \%)$ y que tan solo el $5,09 \%$ se corresponde con contenidos corporativos.
} 


\subsubsection{Stories más allá de Instagram}

Instagram es la red social en la que más marcas y usuarios usan stories, puesto que la posterior asimilación del modelo por parte de Facebook o WhatsApp no refleja, por el momento, una aceptación similar entre los usuarios $^{4}$. Hasta principios de 2018 las historias de Facebook solo estaban disponibles para los perfiles personales, a pesar de que su porcentaje de uso $(5,09 \%)$ distaba mucho del de Instagram $(88,85 \%)$ (Sixto, 2017). Ahora ya es posible emplearlas en la comunicación organizacional realizada a través de Facebook, aunque todavía está pendiente comprobar qué aceptación tendrán por parte de las audiencias. Lo que está claro es que se trata de un plus, un elemento de valor de añadido para la comunicación corporativa, que supone un canal más en la comunicación organización-público, pero que, en ningún caso, colisiona o merma los modelos implementados en Instagram.

La otra red social en la que es posible el desarrollo de stories sigue siendo Snapchat, la red en que se originaron. Sin embargo, el modelo que otros imitaron ha alcanzado más popularidad que el primitivo y, de hecho, Snapchat es una red que cuenta con un número muy inferior de usuarios -255 millones(Galeano, 2018), a pesar de que en 2016 incrementó un 7\% su conocimiento espontáneo por parte de los usuarios con respecto al año anterior (IAB, 2017). Con todo, si comparamos el dato con Instagram encontramos que esta última incrementó su notoriedad en los últimos tres años y se situó como la tercera red más presente en la mente de los internautas. De igual modo, el mismo estudio (IAB, 2017) señala que el uso/visita de Instagram en 2016 aumentó en un $34 \%$ frente al $5 \%$ de Snapchat.

\footnotetext{
${ }^{4}$ En el mismo estudio anterior (Sixto, 2017) se comprobó que de los usuarios que emplean stories en su comunicación habitual el $88,85 \%$ lo hace en Instagram (5,09\% en Facebook y 6,05\% en WhatsApp). Por otra parte, también se comprobó que el $32,29 \%$ de los usuarios de Instagram utiliza stories en su comunicación habitual.
} 


\section{METODOLOGÍA}

La investigación presentada arranca de la revisión del estado del arte para determinar (1) la idoneidad del contenido audiovisual en la comunicación profesional de marcas personales y comerciales y (2) el uso concreto de Instagram en las estrategias de community management.

Una vez explorado lo que la comunidad académica ha aportado a la cuestión, la contribución de este trabajo consiste en (1) localizar las cuentas de Instagram más influyentes en el mundo y en España y (2) determinar los patrones comunes que presentan esas cuentas y que, en consecuencia, establecen los parámetros a considerar para evaluar la influencia de los perfiles en esa red social. En ambos casos se valoran elementos de carácter cuantitativo referentes a número de seguidores y seguidos, y número de publicaciones e interacciones sociales, pero también factores de carácter cualitativo vinculados al análisis del contenido como el empleo de formatos snap y storyboards.

\section{RESULTADOS}

Presentamos a continuación la relación de las diez cuentas de Instagram con mayor número de seguidores en el mundo y, por tanto, con mayor capacidad de influencia sobre las audiencias. La media de seguidores es de 123,49 millones, mientras que el número de perfiles a los que siguen esas cuentas es tan solo de 250. La media de publicaciones es de 3.087,1 por perfil: 
Tabla 1: Top 10 de las cuentas de Instagram más seguidas en el mundo

\begin{tabular}{|c|c|c|c|c|c|}
\hline Puesto & Perfil & Profesión & Seguidores & Seguidos & Publicaciones \\
\hline 1 & $\begin{array}{l}\text { Instagram } \\
\text { @instagram }\end{array}$ & Corporativa & 232,7 millones & 193 & 5.012 \\
\hline 2 & $\begin{array}{l}\text { Selena Gómez } \\
@ \text { selenagomez }\end{array}$ & Cantante & 133,9 millones & 37 & 1.409 \\
\hline 3 & $\begin{array}{c}\text { Cristiano Ronaldo } \\
@ \text { cristiano }\end{array}$ & Futbolista & 121,4 millones & 389 & 2.187 \\
\hline 4 & $\begin{array}{l}\text { Ariana Grande } \\
@ \text { arianagrande }\end{array}$ & Cantante & 117,9 millones & 1.340 & 3.376 \\
\hline 5 & $\begin{array}{l}\text { Beyoncé } \\
\text { @beyonce }\end{array}$ & Cantante & 111,9 millones & 0 & 1.585 \\
\hline 6 & $\begin{array}{l}\text { Kim Kardashian } \\
\text { @kimkardashian }\end{array}$ & Celebrity & 108,3 millones & 121 & 4.168 \\
\hline 7 & $\begin{array}{l}\text { Taylor Swift } \\
@ \text { taylorswift }\end{array}$ & Cantante & 106,5 millones & 0 & 104 \\
\hline 8 & $\begin{array}{l}\text { Kylie Jenner } \\
@ \text { kyliejenner }\end{array}$ & Modelo & 104,5 millones & 127 & 5.353 \\
\hline 9 & $\begin{array}{l}\text { The Rock } \\
\text { @therock }\end{array}$ & Actor y luchador & 100,6 millones & 204 & 3.378 \\
\hline 10 & $\begin{array}{l}\text { Justin Bieber } \\
\text { @justinbieber }\end{array}$ & Cantante & 97,2 millones & 89 & 4.299 \\
\hline
\end{tabular}

Fuente: elaboración propia consultando Instagram

De las diez cuentas que configuran el top 10 mundial solo una (10\%) se corresponde con un perfil de empresa. Se trata de @instagram, la cuenta más seguida del mundo, mientras que el resto $(90 \%)$ corresponden a marcas personales cuyos niveles de popularidad están consolidados fuera de las redes sociales y son anteriores a la explotación en redes de la marca personal.

Es necesario extender la lista hasta la posición 20 para encontrar dos cuentas organizacionales: la entidad sin ánimo de lucro National Geographic (puesto 14) y la marca de ropa deportiva Nike (puesto 16): 
Tabla 2: Top 20 de las cuentas de Instagram más seguidas en el mundo

\begin{tabular}{|c|c|c|c|c|c|}
\hline Puesto & Perfil & Profesión & Seguidores & Seguidos & Publicaciones \\
\hline 11 & $\begin{array}{l}\text { Neymar JR } \\
\text { @neymarjr }\end{array}$ & Futbolista & 89,8 millones & 724 & 4.089 \\
\hline 12 & $\begin{array}{l}\text { Kendall Jenner } \\
\text { @kendalljenner }\end{array}$ & Modelo & 87,7 millones & 191 & 2.895 \\
\hline 13 & $\begin{array}{l}\text { Leo Messi } \\
\text { @leomessi }\end{array}$ & Futbolista & 87,7 millones & 200 & 290 \\
\hline 14 & $\begin{array}{l}\text { NatGeo } \\
\text { @natgeo }\end{array}$ & Corporativa & 86,1 millones & 123 & 16.485 \\
\hline 15 & $\begin{array}{l}\text { Nicki Minaj } \\
\text { @nickiminaj }\end{array}$ & Cantante & 85,8 millones & 1.023 & 5.179 \\
\hline 16 & $\begin{array}{l}\text { Nike } \\
@ \text { @ike }\end{array}$ & Corporativa & 76,2 millones & 136 & 915 \\
\hline 17 & $\begin{array}{l}\text { Miley Cyrus } \\
@ \text { mileycyrus }\end{array}$ & Cantante & 74,4 millones & 576 & 6.747 \\
\hline 18 & $\begin{array}{c}\text { Khloe Kardashian } \\
\text { @khloekardashian }\end{array}$ & Celebrity & 73,1 millones & 143 & 3.243 \\
\hline 19 & $\begin{array}{c}\text { Jennifer López } \\
\text { @jlo }\end{array}$ & Cantante & 72,8 millones & 1.057 & 2.231 \\
\hline 20 & $\begin{array}{l}\text { Katy Perry } \\
\text { @katyperry }\end{array}$ & Cantante & 68,5 millones & 344 & 892 \\
\hline
\end{tabular}

Fuente: elaboración propia consultando Instagram

Al acotar geográficamente el análisis al caso de España y analizar las cuentas españolas más seguidas la situación se repite y solo encontramos marcas personales (100\%) en el top 10. En este caso la media de seguidores es de 3,18 millones y la de cuentas a las que siguen de 445,75 , mientras que la media de publicaciones es de 1.878,91 por perfil.

Sin embargo, en España encontramos una particularidad que no se reproduce al examinar las cuentas desde una óptica globlal: cuatro de las cuentas que integran el top $10(40 \%)^{5}$ no gozaban de popularidad fuera de las redes sociales 0 , dicho de otro modo, han sido las redes sociales las que les han permitido hacerse populares y lograr la capacidad de influencia que poseen hoy en día (puestos 1, 3, 4 y 8b). En efecto, el primer puesto de las cuentas

\footnotetext{
${ }^{5}$ El porcentaje se reduce al 33,33\% si consideramos 12 posiciones en lugar de 10.
} 
españolas con más seguidores lo ocupa Rubén Doblas, El Rubius, un joven anónimo que acaba de cumplir seis años de reinado en YouTube y cuenta con 27 millones de followers en su canal:

Tabla 3: Top 10 de las cuentas españolas de Instagram más seguidas ${ }^{6}$

\begin{tabular}{|c|c|c|c|c|c|}
\hline Puesto & Perfil & Profesión & Seguidores & Seguidos & Publicaciones \\
\hline 1 & El Rubius @elrubiuswtf & Youtuber & 7,7 millones & 158 & 478 \\
\hline 2 & Rafa Nadal @rafaelnadal & Tenista & 4,4 millones & 41 & 408 \\
\hline $3 a$ & Guillermo Díaz@willyrex & Youtuber & 2,9 millones & 76 & 513 \\
\hline $3 b$ & $\begin{array}{c}\text { Penélope Cruz } \\
\text { @penelopecruzoficial }\end{array}$ & Actriz & 2,9 millones & 244 & 580 \\
\hline 4 & $\begin{array}{c}\text { Samuel de Luque } \\
\text { @vegetta777 }\end{array}$ & Youtuber y blogger & 2,8 millones & 0 & 149 \\
\hline 5 & $\begin{array}{l}\text { Pablo Alborán } \\
\text { @pabloalboran }\end{array}$ & Cantante & 2,7 millones & 1.708 & 2.349 \\
\hline 6 & $\begin{array}{l}\text { Alejandro Sanz } \\
\text { @alejandrosanz }\end{array}$ & Cantante & 2,6 millones & 844 & 1.471 \\
\hline 7 & $\begin{array}{l}\text { Jorge Cremades } \\
\text { @jorgescremades }\end{array}$ & Actor & 2,4 millones & 240 & 800 \\
\hline $8 a$ & $\begin{array}{l}\text { Abraham Mateo } \\
\text { @abrahammateo }\end{array}$ & Cantante & 2,2 millones & 152 & 1.553 \\
\hline $8 b$ & $\begin{array}{c}\text { Aída Domenech } \\
\text { @dulceida }\end{array}$ & $\begin{array}{c}\text { Blogger, } \\
\text { instagramer y } \\
\text { youtuber }\end{array}$ & 2,2 millones & 721 & 6.957 \\
\hline 9 & $\begin{array}{c}\text { Paula Echevarría } \\
\text { @pau eche }\end{array}$ & Actriz & 2,1 millones & 806 & 6.249 \\
\hline 10 & $\begin{array}{l}\text { David Bisbal } \\
\text { @davidbisbal }\end{array}$ & Cantante & 2 millones & 359 & 1.040 \\
\hline
\end{tabular}

Fuente: elaboración propia consultando Instagram

\footnotetext{
${ }^{6}$ Los puestos 3 y 8 aparecen duplicados porque el número de seguidores es idéntico entre las posiciones a y b. Se utiliza el orden alfabético para determinar la primera posición dentro del subconjunto.
} 
Parece evidente que el número de seguidores de una cuenta de Instagram es el primer elemento que determina su nivel de influencia. Influir exige necesariamente sujetos sobre quien poder hacerlo y, en consecuencia, ningún perfil puede ser influyente sin un número elevado de seguidores. Pero, aparte del número de followers, las cuentas influyentes tanto en el ámbito mundial como español presentan una serie de patrones comunes:

a) El número de seguidores siempre es muy superior al número de seguidos, de tal forma que a mayor nivel de follow back menor capacidad de influencia. En el conjunto de las cuentas más influyentes en el mundo sus titulares solo siguen al $0,0002 \%$ de sus seguidores, mientras que en el caso español el porcentaje se sitúa en el 0,014\%. Algunas cuentas llevan este principio hasta el extremo y, así, en el top 10 mundial encontramos dos casos (@beyonce y @taylorswift) con 0 seguidores y en el top 10 español uno (@vegetta777).

b) Ninguna de las cuentas incluidas en los tops 10 representa una presencia testimonial, sino que son cuentas activas desde el punto de vista de las publicaciones $(3.087,10$ en el top mundial y $1.878,91$ en el español), aunque eso no implica necesariamente que se trate de cuentas concebidas como espacios dialógicos con los followers, puesto que se aprecian indicios de canales unidireccionales en los que las marcas exponen y las audiencias reaccionan y valoran.

c) Adaptación permanente a la evolución técnica y tecnológica y consecuente adopción de los nuevos modelos de storytelling como es el caso de las historias.

\section{CONCLUSIONES}

Las marcas personales son más influyentes en Instagram que las marcas comerciales u organizacionales. De hecho, entre las diez cuentas con mayores niveles de influencia en los ámbitos mundial y español solo se localiza una 
cuenta corporativa, la del propio Instagram, que a su vez es la cuenta más seguida en el mundo. Por tanto, la influencia en Instagram está directamente vinculada a la explotación de marcas personales en detrimento de las marcas organizacionales, a las que todavía les queda un largo camino para equiparse con las primeras.

Conscientes de esta situación, las organizaciones recurren a los influencers que trabajan su marca personal para que actúen como presciptores y embajadores de los productos que ofrecen al mercado, pues la capacidad de autoridad de estas figuras sobre las audiencias confiere a los productos confianza y credibilidad. La gran mayoría de esos influencers (100\% en el nivel mundial y $60 \%$ en España) son personajes públicos que son conocidos por su profesión y/o apariciones en los medios de comunicación tradicionales, aunque en el caso de España un $40 \%$ han alcanzado la popularidad exclusivamente en las redes sociales. Es el caso de los youtubers y bloggers de moda que complementan su trabajo en otros espacios sociales con la presencia en Instagram.

El nivel de influencia de una cuenta en Instagram está determinado, en primer lugar, por el número de seguidores, pero a ello se añaden otros tres patrones que comparten todas las cuentas influyentes: (1) una ratio inapreciable de follow back a los seguidores de la cuenta; (2) la frecuencia de publicación y la cantidad de publicaciones; y (3) el uso de todos los recursos y potencialidades que ofrece la red social para la implementación del storytelling, no solo el almacenaje de fotografías y vídeos, sino también la utilización habitual de contenido autodestruible.

Desde la perspectiva de quien gestiona la cuenta, a estos medidores se ha de sumar inexcusablemente la evaluación analítica de resultados, que permitirá comprobar la evolución de la cuenta y la adecuación de los resultados a los objetivos establecidos. Se considerarán, pues, el número de reproducciones de 
los vídeos y el tiempo promedio de visualización, el número de interacciones, los enlaces externos recibidos, las menciones en otros sitios web o las visitas redigiridas hacia la web corporativa o hacia las redes sociales.

No podemos establecer una conexión directa entre influencia y engagement, pues a pesar de que todas las cuentas analizadas son influyentes solo algunas representan algo más que espacios de soliloquio. Si bien es cierto que se concede protagonismo a los seguidores, no siempre se interactúa con ellos. $\mathrm{Ni}$ la galería de publicaciones ni el storytelling de las historias deben convertirse en un tablón de anuncios que solo hable de la marca, sino que la tendencia es implicar a los públicos cada vez más, de manera que el power to the people cree incluso rutinas de publicación que acostumbren a las audiencias a contenidos seriados y a ciertos tópicos que se repiten y que acaban personalizando la cuenta.

Si los públicos no se tienen en cuenta y las publicaciones se realizan de manera robótica, Instagram puede convertir los hashtags utilizados en no detectables. Es lo que se conoce como shadow ban, un fenómeno que afecta sobre todo a Twitter e Instagram. Por ello, un uso responsable de los hashtags será lo que potenciará el alcance de las publicaciones gracias a las búsquedas que realizan los usuarios. De lo contrario, Instagram puede frenar el alcance y mostrar las publicaciones en las búsquedas por hashtag solo a los seguidores, lo que limita las posibilidades de alcanzar nuevos followers y reduce el número de comentarios y likes, algo que repercutiría de manera directa en los niveles de autoridad e influencia. 


\section{REFERENCIAS BIBLIOGRÁFICAS}

Abidin, C. (2016). Visibility labour: Engaging with Influencers' fashion brands and \#OOTD advertorial campaigns on Instagram. Media International Australia, 161(1), 86-100. DOI: https://doi.org/10.1177/1329878X16665177

Agustina, M. \& Steinberg, L. (2017). Youtuber discursivity: Affection, narratives and socialization strategies in Internet communities. Revista Mediterránea de Comunicación, $\quad 8(2), \quad 171-188 . \quad$ DOI: https://doi.org/10.14198/MEDCOM2017.8.2.12

Alp, Z. Z. \& Oguducu, S. G. (2018). Identifying topical influencers on twitter based on user behavior and network topology. Knowledge-Based Systems, 141, 211-221. DOI: https://doi.org/10.1016/j.knosys.2017.11.021

De Veirman, M., Cauberghe, V. \& Hudders, L. (2017). Marketing through Instagram influencers: the impact of number of followers and product divergence on brand attitude. International Journal of Advertising, 36(5), 798828. DOI: https://doi.org/10.1080/02650487.2017.1348035

Engelstaetter, B. \& Ward, M. R. (2018). Strategic timing of entry: evidence from video games. Journal of Cultural Economics, 42(1), 1-22. DOI: https://doi.org/10.1007/s10824-016-9276-7

Galeano, S. (2018). Cuáles son las redes sociales con más usuarios del mundo (2018). Marketing4ecommerce. Recuperado el 15 de febrero de 2018 de https://marketing4ecommerce.net/cuales-redes-sociales-mas-usuarios-mundo2018/

García, A., López-de-Ayala, M.C. \& Catalina, B. (2013). Hábitos de uso en Internet y en las redes sociales de los adolescentes españoles. Comunicar 2013, XXI(41), 195-204. DOI: https://doi.org/10.3916/C41-2013-19 
Garmendia, M., Garitaonandia, C., Martínez, G. \& Casado, M. (2012). Riesgos y seguridad en Internet: los menores españoles en el contexto europeo. Quaderns del CAC 38, XV (1), 37-44.

Godwin, H. T., Khan, M. \& Yellowlees, P. (2017). The Educational Potential of YouTube. Academic Psychiatry, 41(6), 823-827. DOI: https://doi.org/10.1007/s40596-017-0809

Gruner, R. L. \& Power, D. (2018). To integrate or not to integrate? Understanding B2B social media communications. Online Information Review, 42(1), 73-92. DOI: https://doi.org/10.1108/OIR-04-2016-0116

Gupta, H., Lam, T.; Pettigrew, S. \& Tait, R. (2018). Alcohol marketing on YouTube: exploratory analysis of content adaptation to enhance user engagement in different national contexts. BMC Public Health, 18, artículo 14. DOI: https://doi.org/10.1186/s12889-018-5035-3

Horta, P. M., Rodrigues, F. T. \& Dos Santos, L. C. (2018). Ultra-processed food product brands on Facebook pages: highly accessed by Brazilians through their marketing techniques. Public Health Nutrition, 1-5. DOI: https://doi.org/10.1017/S1368980018000083

IAB (2017). Estudio Anual de Redes Sociales. Recuperado el 12 de febrero de 2018 http://iabspain.es/wpcontent/uploads/iab_estudioredessociales_2017_vreducida.pdf

Mamani, M. J., do Nascimento, D. E., Rocha, S. H. \& Santoyo, A. H. (2017). Analysis of addiction test applied to adolescents, users of digital social media. Revista Espacios, 38(24). Recuperado de http://www.revistaespacios.com/a17v38n24/17382406.html

Mullo-Lopez, A.-H. \& Yaguana-Romero, H.-A. (2017). The prosumer in the construction of the radio speech: analysis of the case of the Ecuadorian radios 
of Cotopaxi and Tungurahua. Revista Mediterránea de Comunicación, 8(2), 241-260. DOI: https://doi.org/10.14198/MEDCOM2017.8.2.15

Pathcin, J. W., \& Hinduja, S. (2010). Trends in Online Social Networking: Adolescent Use of MySpace over Time. New Media \& Society, 12(2), 197-216. DOI: https://doi.org/ 10.1177/1461444809341857

Planells, J. A. (2017). Video games and the crowdfunding ideology: From the gamer-buyer to the prosumer-investor. Journal of Consumer Culture, 17(3), 620-638. DOI: https://doi.org/10.1177/1469540515611200

Rodríguez, M. I., Paino, A., Ruiz, Y. \& Jiménez, L. (2017). Cambios en los modelos persuasivos: la nueva publicidad en tiempo real a través de estrategias narrativas transmedia. Estudio de caso de la campaña publicitaria de Tous, Tender Stories. adComunica. Revista Científica de Estrategias, Tendencias e Innovación en Comunicación, 14, 27-50.

Seyfi, M. \& Soydas, A. U. (2017). Instagram Stories from the perspective of narrative transportation theory. Turkish Online Journal of Design Art and Communication, 7(1), 47-60. DOI: https://doi.org/10.7456/10701100/005

Sixto, J. (2017). Os contidos autodestruibles, unha nova forma de storytelling para a comunicación organizacional. En Ledo, M.; Campos, F.; Toural, C.; Rúas, X.; Rodríguez, A. I.; Costa, C.; Salgueiro, M. \& Rodríguez, M. (eds.) (2017). Actas do I Internacional de AGACOM. Alén das Fronteiras: Redes na Diversidade, 385-398. Santiago de Compostela: Universidade de Santiago de Compostela. 